\title{
Investigation of deformation behavior of PETG-FDM-printed metamaterials with pantographic substructures based on different slicing strategies
}

Composites and Advanced Materials Volume 30: I-13 (C) The Author(s) 2021 Article reuse guidelines: sagepub.com/journals-permissions DOI: $10.1177 / 26349833211016477$ journals.sagepub.com/home/acm (SAGE

\author{
Arda Özen', Gregor Ganzosch ${ }^{2} \oplus$, Emilio Barchiesi ${ }^{3}$, \\ Dietmar W Auhl' and Wolfgang $H$ Müller ${ }^{2}$
}

\begin{abstract}
Based on the progress and advances of additive manufacturing technologies, design and production of complex structures became cheaper and therefore rather possible in the recent past. A promising example of such complex structure is a socalled pantographic structure, which can be described as a metamaterial consisting of repeated substructure. In this substructure, two planes, which consist of two arrays of beams being orthogonally aligned to each other, are interconnected by cylinders/pivots. Different inner geometries were taken into account and additively manufactured by means of fused deposition modeling technique using polyethylene terephthalate glycol (PETG) as filament material. To further understand the effect of different manufacturing parameters on the mechanical deformation behavior, three types of specimens have been investigated by means of displacement-controlled extension tests. Different slicing approaches were implemented to eliminate process-related problems. Small and large deformations are investigated separately. Furthermore, 2D digital image correlation was used to calculate strains on the outer surface of the metamaterial. Two finiteelement simulations based on linear elastic isotropic model and linear elastic transverse isotropic model have been carried out for small deformations. Standardized extension tests have been performed on 3D-printed PETG according to ISO 527-2. Results obtained from finite-element method have been validated by experimental results of small deformations. These results are in good agreement with linear elastic transverse isotropic model (up to about $\varepsilon_{x x}=1.2 \%$ of axial elongation), though the response of large deformations indicates a nonlinear inelastic material behavior. Nevertheless, all samples are able to withstand outer loading conditions after the first rupture, resulting in resilience against ultimate failure.
\end{abstract}

\section{Keywords}

polymeric metamaterial, pantographic sheet, 3D-printing/fused-deposition-modeling, experiments, digital image correlation

\section{Introduction}

Rapid prototyping enables the manufacturing of extraordinary materials resulting in structures with very complex geometries. Those tailor-made structures, whose special properties such as high stiffness or large deformation depend on the microscopic constituents, ${ }^{1,2}$ are known as so-called architectured materials or metamaterials, inter alia. Hence, the mechanical deformation behavior of such a metamaterial is very sensitive to the substructure, not just to the global structure.

A pantographic structure (PS) can be treated as a metamaterial consisting of two orthogonal arrays of beams that are interconnected by pivots/cylinders. ${ }^{3-7}$ An example of

\footnotetext{
'Department of Material Science and Engineering, Technische Universität Berlin, Department of Polymer Materials Science and Technologies, Institute of Material Science and Technology, School 3, Berlin, Germany

${ }^{2}$ Department of Mechanical Engineering, Technische Universität Berlin, Department of Continuum Mechanics and Materials Theories, Institute of Mechanics, School 5, Berlin, Germany

${ }^{3}$ Department of Information Engineering, Computer Science and Mathematics, International Research Center for Mathematics \& Mechanics of Complex Systems (M\&MoCS), University of L'Aquila, Berlin, Germany
}

Date received: 8 September 2020; accepted: 13 April 202I

Corresponding author:

Gregor Ganzosch, Technische Universität Berlin, Continuum Mechanics and Materials Theory, Institute of Mechanics, School 5, Sekr. MS2, Einsteinufer 5, 10587 Berlin, Germany.

Email: ganzosch@tu-berlin.de 


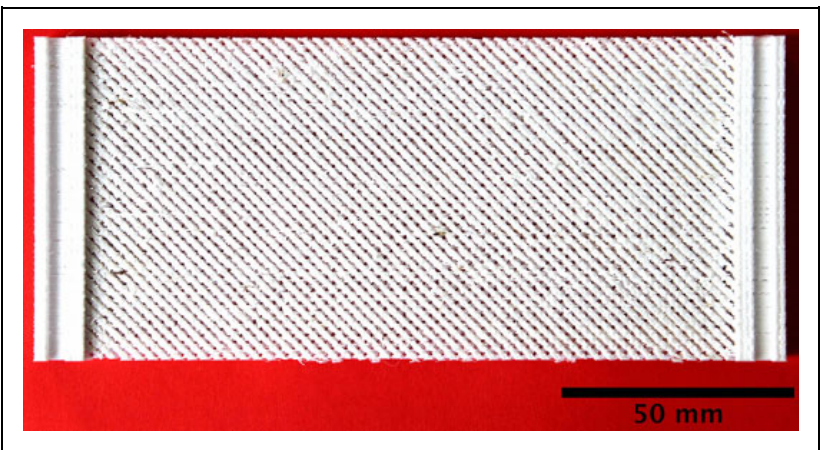

Figure I. The 3D-printed metamaterial made of PETG: Front view of a so-called pantographic structure. PETG: polyethylene terephthalate glycol.

a PS made out of polyethylene terephthalate glycol (PETG) is shown in Figure 1. To design such a metamaterial for special applications relevant in the industry sector, predictions of their structural performances are crucial. A possibility to achieve deformation predictions is to make use of the finite-element method (FEM). This involves a very detailed mesh of the whole structure, ${ }^{8}$ which results in high computational costs. The reason for these computational costs is the high number of unknowns in a classic numerical simulation, which is based on the Cauchy-Boltzmann continuum. However, higher gradient theories are able to overcome these limits by homogenizing discrete structures toward the equivalent Cauchy continua. ${ }^{2,9-12}$ Thus, PS is a very auspicious example showing this property because it can be modeled by means of higher gradients (e.g. second gradient continuum model $^{4}$ ). By formulating the constitutive laws, new and unknown material parameters show up. To determine these newly appeared parameters, experiments have to be performed. ${ }^{13-16}$

In this research, uniaxial tensile tests were conducted on 3D-printed pantographic metamaterials made out of PETG. Three different variations of pantographics with differently sized substructures are investigated and discussed. Results of extension tests, specifically the stress-strain relations (in the linear elastic region up to about $1.5 \%$ strain), will be compared to results obtained from (homogenized) FEM calculations based on the open-source library of FEniCS. ${ }^{17}$ Both small and large deformations are recorded and evaluated by means of two-dimensional digital image correlation (DIC). Furthermore, three different slicing procedures have been implemented into the additive manufacturing process. The effect of each slicing approach on the deformation behavior is investigated. Notice that the motivation of this work is not only to perform experimental characterizations but also to enable the evaluation of higher gradient simulation models for the determination of new higher gradient parameters in future investigations.

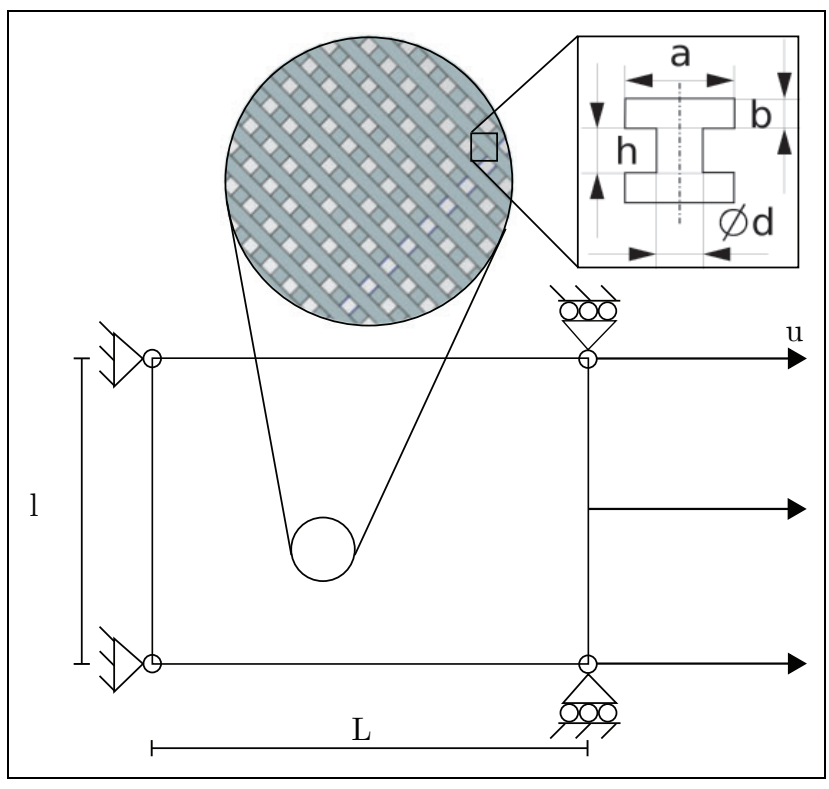

Figure 2. Schematic of displacement-controlled extension test ( $u=$ displacement) performed on PS $(L=$ width, $I=$ height, and $t$ $=$ depth) including details of the unit cell $(a, b=$ width and height of beam, $d=$ diameter of interconnected pivot/cylinder, and $h=$ height of pivot/cylinder). PS: pantographic structure.

\section{Materials and methods}

Metamaterials with pantographic substructures were 3D printed using fused deposition modeling (FDM) technique at Technische Universität Berlin, Institute of Mechanics, Chair of Stability and Failure of Functionally Optimized Structures. Details of additive manufacturing are introduced in "Manufacturing" section. To determine linear elastic material parameters of PETG, which are used as input for the numerical simulations, uniaxial, standardized extension tests of five tensile specimens have been performed at Technische Universität Berlin, Institute of Material Science and Technology, Department of Polymer Materials Science and Technologies. Furthermore, quasistatic extension tests and 2D DIC measurements applied to six PSs have been performed at Technische Universität Berlin, Institute of Mechanics, Department of Continuum Mechanics and Materials Theories. The schematic of experimental setup including PS is shown in Figure 2. Standardized extension tests performed on standardized tensile specimens and experiments performed on PSs are described in "Experimental setup" section. Linear elastic numerical simulation models are introduced and explained in "Finite-element method" section.

\section{Manufacturing}

PSs, composed out of rectangular beams (with a quadratic cross-section $A=a * b=a^{2}$ ) and cylindrical pivots/joints (with a diameter $d$ and height $h$, see Figure 1 and detailview of Figure 2), have been manufactured by the FDM 
Table I. FDM process parameters and PETG material parameters (given by Material 4 Print $\mathrm{GmbH} \&$ Co. KG).

\begin{tabular}{lcc}
\hline Printing parameters & Value & Unit \\
\hline Layer thickness & 0.2 & $\mathrm{~mm}$ \\
Layer width & 0.4 & $\mathrm{~mm}$ \\
Print speed & 55 & $\mathrm{~mm} / \mathrm{s}$ \\
Initial layer speed & 40 & $\mathrm{~mm} / \mathrm{s}$ \\
Print acceleration & 4000 & $\mathrm{~mm} / \mathrm{s}^{2}$ \\
Print temperature & 250 & ${ }^{\circ} \mathrm{C}$ \\
Print temperature initial layer & 255 & ${ }^{\circ} \mathrm{C}$ \\
Final printing temperature & 240 & ${ }^{\circ} \mathrm{C}$ \\
Bed temperature & 70 & ${ }^{\circ} \mathrm{C}$ \\
Material properties & & \\
Mass density & 1.27 & $\mathrm{~g} / \mathrm{cm}$ \\
Elongation at break & 70 & $\%$ \\
Tensile strength & 53 & $\mathrm{MPa}$ \\
Flexural modulus & 2150 & $\mathrm{MPa}$ \\
Melting point & $200-230$ & ${ }^{\circ} \mathrm{C}$ \\
Heat distortion temperature & 74 & ${ }^{\circ} \mathrm{C}$ \\
\hline
\end{tabular}

printer (Ultimaker 3 Extended, Ultimaker B.V., Geldermalsen, The Netherlands). The 3D printer was controlled by the device-own software Cura 4.2 (Ultimaker B.V.), in which the process parameters such as temperature, slicing speed, or layer thickness are defined (for further information, see Table 1). PETG (Material 4 Print $\mathrm{GmbH} \&$ Co. $\mathrm{KG}$, Bad Oeynhausen, Germany) filaments were used as a raw material. Additionally, polyvinyl acetate (PVA) filaments (Ultimaker B.V.) were employed as a support structure in the printing process and solved afterward. Process parameters and material properties are provided in Table 1. We assume that those manufacturer parameters have been determined by testing mold specimens, and hence, their porosity should be nearly zero. Therefore, we consider them as upper parameter thresholds for 3D-printed polymers.

Due to different solidification characteristics, temperature profile, and lack of molecular diffusion, the mechanical resistance of interfiber bonding regions against deformation is not as good as the inner one. ${ }^{18}$ In samples printed with layer orientations of $g=90^{\circ}$ with respect to the lateral plane of the sample, tensile forces mostly affect the interfiber regions. Therefore, they should have weaker mechanical properties (e.g. against failure). However, these data are mostly gained using standardized tensile test specimens. But, it was shown in the literature ${ }^{18}$ that manipulated samples with slightly different shapes/geometries show the same tendency. It should also be mentioned that the inner structure of metamaterials such as PSs is much more challenging and makes it very difficult to estimate the effect of printing orientations on the mechanical deformation behavior. Notes of state-of-theart on this topic are very rare in the literature. To investigate that phenomenon in more detail, we also print and compare samples consisting of different layer orientations $\left(0^{\circ}\right.$ and $90^{\circ}$ with respect to the axial/lateral printing direction). Therefore, it is necessary to ensure a unidirectional (UD) printing procedure. However, slicer software implements printing algorithms that disturb the manufacturing of a UD structure, for example, by exceeding inner travel lines. ${ }^{19}$ Such a disruption may cause material accumulation resulting in local stress peaks and makes a comparative analysis unreliable. Hence, three different slicing strategies are taken into account and are depicted in Figure 3.

Furthermore, due to the significant influence of the microscopic substructure onto the macroscopic deformation behavior, ${ }^{20-27}$ different kinds of PSs with varied inner geometries (to be more precise with different pivot heights $h$ ) and differently sized outer dimensions (to be more precise with different sample heights $l$ ) but equal material densities were investigated additionally. An overview of sample configurations is given in Table 2.

\section{Experimental setup - standardized extension test}

To determine mechanical deformation parameters (Young's modulus and Poisson's ratio), which will be used for FEM calculations, tensile specimens with $100 \%$ infill were manufactured (without support structure) and parameters listed in Table 1. Their geometries were prepared according to ISO-527-2 (see Figure 4 and Table 3).

Layer orientation is one of the most important process parameters in FDM. The highest tensile properties are generally assumed to be achieved with an orientation of $0^{\circ}$ with respect to the lateral plane of the specimen because, here, loads affect the fibers in longitudinal directions. All five tested standardized tensile specimens were manufactured unidirectionally with an orientation of $0^{\circ}$ (see bottom of Figure 4).

Five uniaxial, displacement-controlled extension tests were performed on the standardized tensile specimens with a quasi-static velocity of $v=2 \mathrm{~mm} / \mathrm{min}$ by means of a Zwick 1446 testing machine (ZwickRoell GmbH \& Co. KG, Ulm, Germany) at Technische Universität Berlin, Institute of Material Science and Technology, Department of Polymer Materials Science and Technologies. Deviceown loading cell and extensometer were employed to measure forces and displacements by means of device own corresponding software (ZwickRoell GmbH \& Co. KG, Ulm, Germany) (see Figure 5).

\section{Experimental setup-extension tests applied to pantographic structures}

Displacement-controlled quasi-static extension tests have been applied to PSs. Schematic setup and front view of the experimental setup of specimen $\mathrm{B} 1$ are shown in Figures 2 and 6, respectively: sample B1 is mounted in the testing device MTS Tytron 250 (MTS Systems Corporation, Eden Prairie, USA). Device-own 


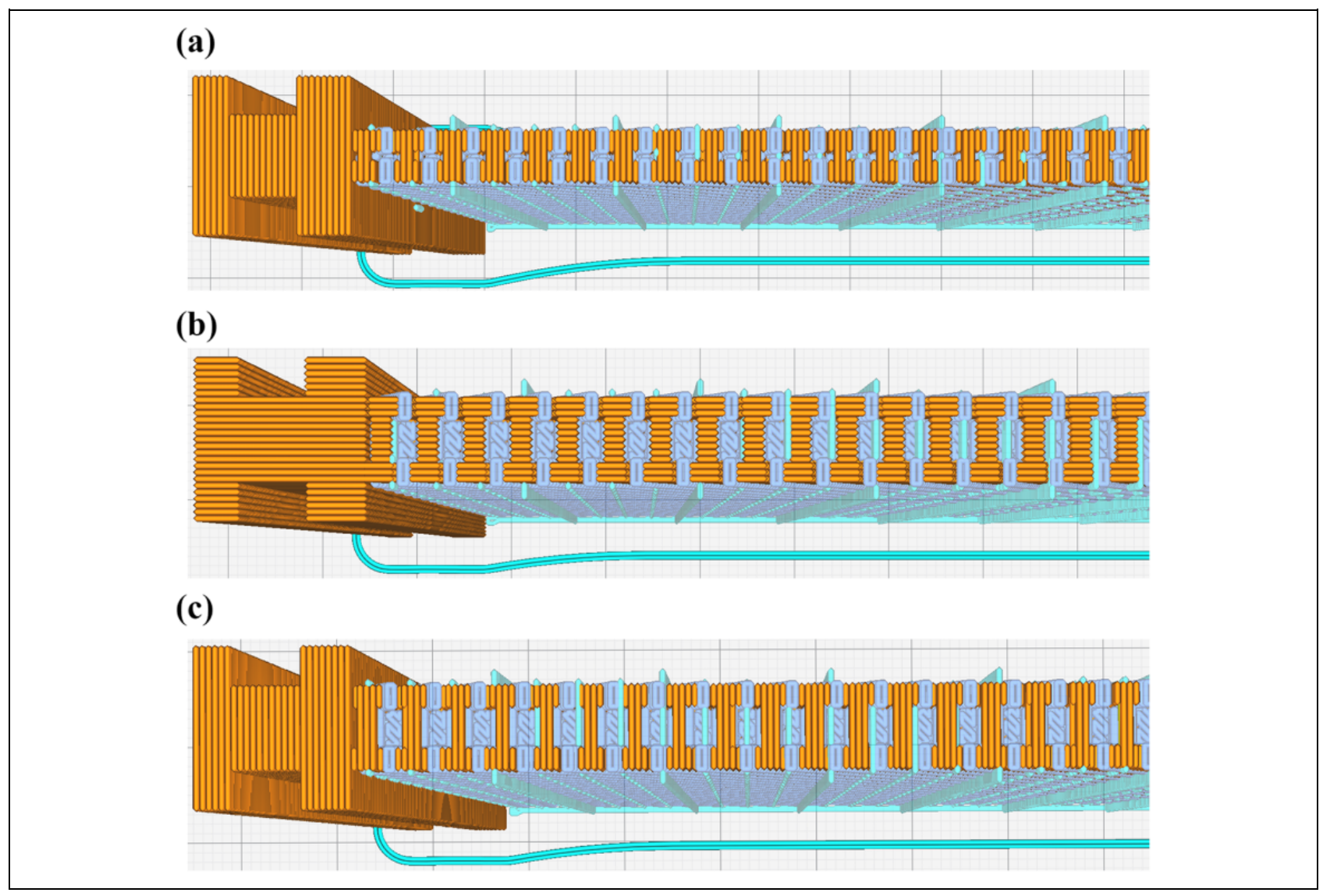

Figure 3. Slicing configurations (Cura): Orange fibers represent infill layers, blue fibers represent support-material layers. All samples are printed upright, not crosswise. (a) Slicing strategy A: Layer direction of $90^{\circ}$ with respect to the axial lateral direction. (b) Slicing strategy B: Layer direction of $0^{\circ}$ with respect to the axial lateral direction. (c) Slicing strategy C: Layer direction of $90^{\circ}$ with respect to the axial lateral direction.

Table 2. Overview of dimensions and layer orientations of all six investigated specimens. ${ }^{\text {a }}$

\begin{tabular}{lrrrrrr}
\hline & Al & A2 & BI & B2 & Cl & C2 \\
\hline$L(\mathrm{~mm})$ & $\mathrm{I} 40$ & $\mathrm{I} 40$ & 140 & $\mathrm{I} 40$ & $\mathrm{I} 40$ & $\mathrm{I} 40$ \\
$l(\mathrm{~mm})$ & 70 & 70 & 70 & 70 & 35 & 35 \\
$t(\mathrm{~mm})$ & 3 & 3 & 5 & 5 & 5 & 5 \\
$a=b(\mathrm{~mm})$ & $\mathrm{I}$ & $\mathrm{I}$ & $\mathrm{I}$ & $\mathrm{I}$ & $\mathrm{I}$ & $\mathrm{I}$ \\
$d(\mathrm{~mm})$ & 0.9 & 0.9 & 0.9 & 0.9 & 0.9 & 0.9 \\
$h(\mathrm{~mm})$ & $\mathrm{I}$ & $\mathrm{I}$ & 3 & 3 & 3 & 3 \\
Slicing strategy & $\mathrm{A}$ & $\mathrm{A}$ & $\mathrm{B}$ & $\mathrm{B}$ & $\mathrm{C}$ & $\mathrm{C}$ \\
Layer orientation & $90^{\circ}$ & $90^{\circ}$ & $0^{\circ}$ & $0^{\circ}$ & $90^{\circ}$ & $90^{\circ}$ \\
\hline
\end{tabular}

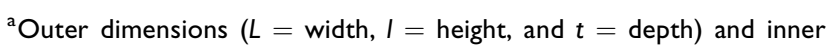
dimensions $(a, b=$ width and height of a beam, $d=$ diameter of a cylinder, $h=$ height of a cylinder) correspond to the schematic in Figure 2. Slicing strategies and layer orientations correspond to Figure 3.

software Stationsmanager V 3.14 controls and monitors the device-own loading cell $(N= \pm 250 \mathrm{~N}$ with an accuracy of $0.2 \%$ ), which is fixed to the left mounting side (see left of Figure 6). Displacement was imposed horizontally $(v=15 \mathrm{~mm} / \mathrm{min})$ through the right mounting side (see right of Figure 6).

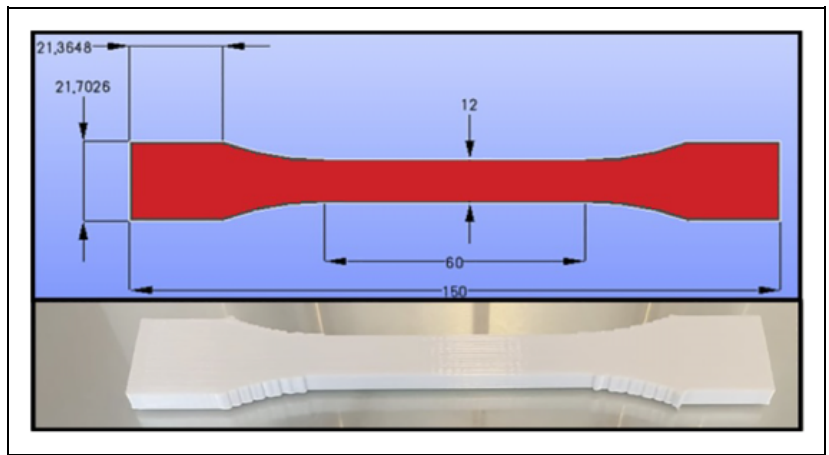

Figure 4. Top: Geometry of standardized tensile specimens according to ISO 527-2. All values are given in mm. Bottom: 3D print of a standardized tensile specimen.

Because of the hydraulic air-film-bearing system, almost friction-less displacement could be achieved. Furthermore, displacement was also measured and evaluated in real time by means of the noninvasive measurement method DIC. This technique is able to calculate displacements and therefore strains on a deformed surface based on recorded pictures taken by a commercial Canon EOS 600D 
Table 3. Specifications of standardized tensile specimens.

\begin{tabular}{lc}
\hline Specimen type & ISO527-2 \\
\hline Tab length & $21.4 \mathrm{~mm}$ \\
Thickness & $6 \mathrm{~mm}$ \\
Length & $150 \mathrm{~mm}$ \\
Angle & R60 \\
Width & $21.7 \mathrm{~mm}$ \\
Gauge length & $60 \mathrm{~mm}$ \\
\hline
\end{tabular}

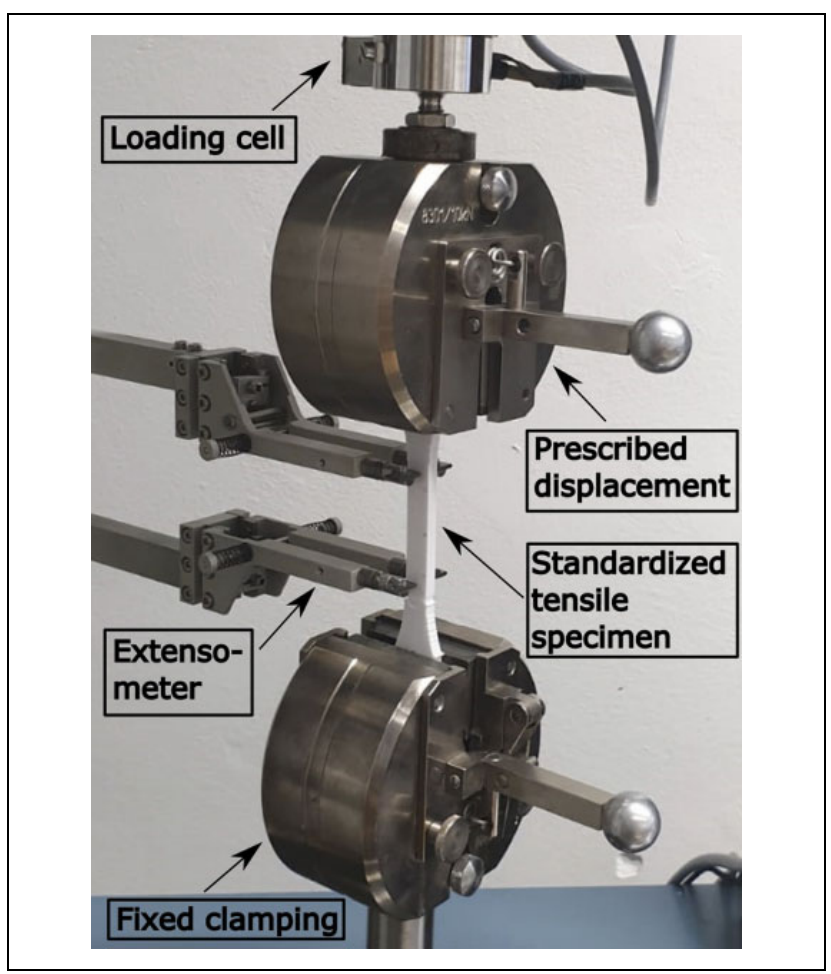

Figure 5. Initial state of a standardized tensile specimen which is clamped into the Zwick 1446 testing device with an extensometer (left) and a loading cell (top). One mounting side is fixed (bottom) while displacement was imposed vertically through the upper mounting side.

camera $(4272 \times 2848$ pixels, 1 picture $/ 2$ s $)$. Before DIC evaluation was performed by means of open-source GOM Correlate software (GOM GmbH, Braunschweig, Germany), a homogeneous speckle pattern had to be applied on top of every sample because of lack of contrast. Figure 7 shows the deformation of whole structure of sample B1 in an extension test from elastic states toward plastic deformation leading to ultimate failure, evaluated by 2D DIC. For more information and details of setup, see the literature by Juritza et al. ${ }^{28}$

\section{Finite-element method}

Two different material models have been taken into account for the numerical investigations on PSs-an

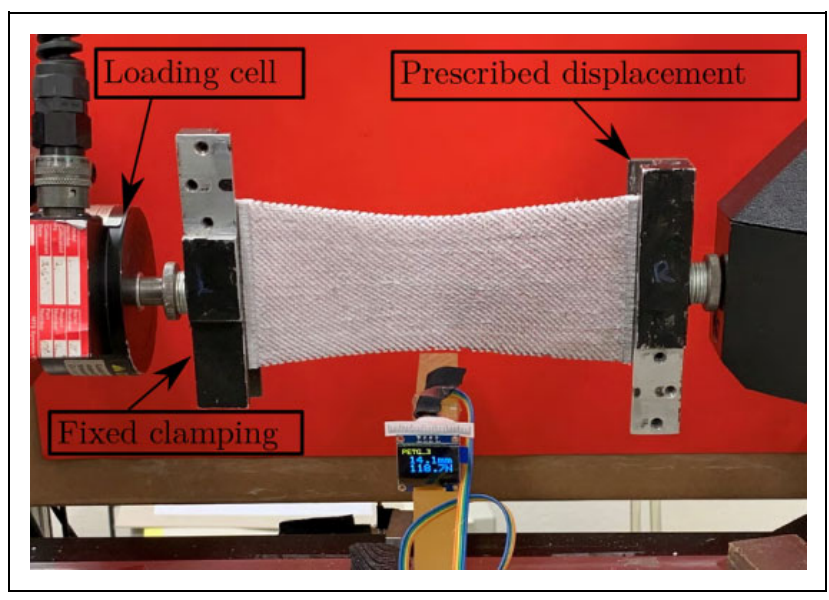

Figure 6. Setup of displacement-controlled extension test performed on speckled specimen BI. Device-own loading cell is fixed in the left clamping jaw while displacement $u$ is imposed horizontally in the right clamping jaw.

isotropic linear-elastic material model and a transverseisotropic, linear-elastic material model. Inverse analysis has been carried out to determine the engineering constants of homogenized transverse elastic stiffness matrix as well as to determine the Young's modulus and Poisson's ratio for FEM input.

All preprocessing steps, that is, computer-aided design (CAD) preparation, boundary conditions, and triangulation were performed using open-source software Salome 9.3. ${ }^{29}$ For a proper CAD preparation of standardized tensile specimens, FDM-printed polymers have been examined by means of a commercial Olympus BX-51 microscope (Olympus Europa SE \& Co. KG, Hamburg, Germany), see Figure 8. Based on dimensions of contact lines between fibers and layers, the process parameters in Table 1 have been employed. CAD models of standardized tensile specimens mainly consist of fibers and voids ${ }^{30}$ while areas of the interface are assumed to have perfect bonding though.

CAD models of PSs have been prepared without any fiber and void features. CAD model of specimen B1 is shown in Figure 9. However, the effect of microstructural dependencies is applied by material models, which are going to be described in the following.

All FEM calculations are performed by means of the open-source codes developed by FEniCS project. ${ }^{17}$ Suitable meshes are obtained after $h$-convergence analysis and their results are shown in Figure 10. Horizontal plateau begins at about 750,000 degrees-of-freedom (DoF) for PS (blue line, Figure 10) and 1,750,000 DoF for standardized tensile specimens (red line, Figure 10), respectively. As the CAD data of a standardized tensile specimen contains very fine and detailed microstructural features, a more discretized mesh is needed for the standardized tensile specimen in contrast to the PSs. Black circles in Figure 10 indicate convergence and show the selected DoF used in the simulations. The $h$-convergence of strain energy ${ }^{31}$ is 


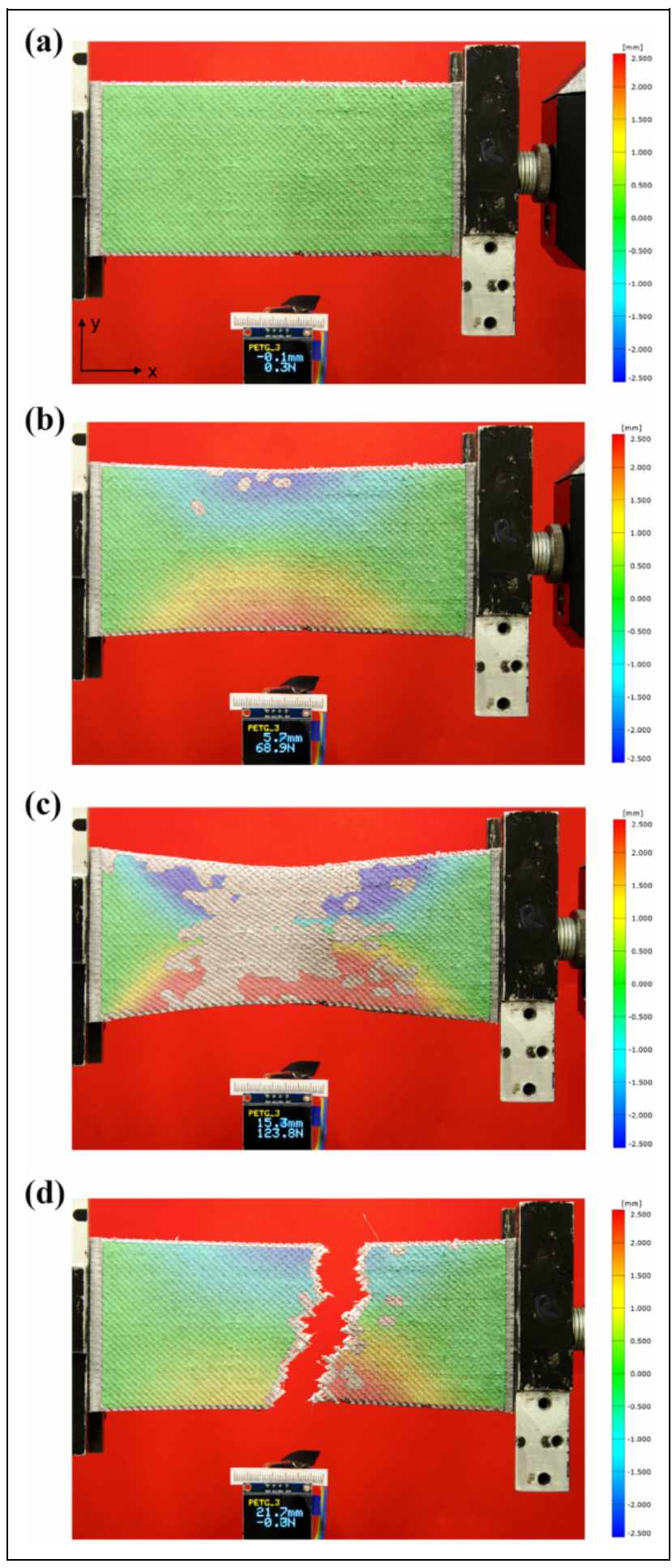

Figure 7. Picture-sequence taken during deformation of sample BI, validated by $2 \mathrm{D}$ DIC (vertical displacement $u_{y y}= \pm 2.5 \mathrm{~mm}$ ).

(a) Initial state before deformation. (b) During deformation: a necking of the specimen can be recognized at an axial elongation of about $\varepsilon_{x x}^{B I}=4.1 \%$. Vertical displacement reaches about $u_{y y}^{B I}= \pm 2.5 \mathrm{~mm}$ at this deformation state. (c) After first failure: at an axial elongation of about $\varepsilon_{x x}^{B I}=10.4 \%$, a pivot breaks locally in the upper left corner of the specimen without resulting in ultimate failure. (d) After ultimate failure: global rupture through the whole specimen in vertical direction (top-down). DIC: digital image correlation.

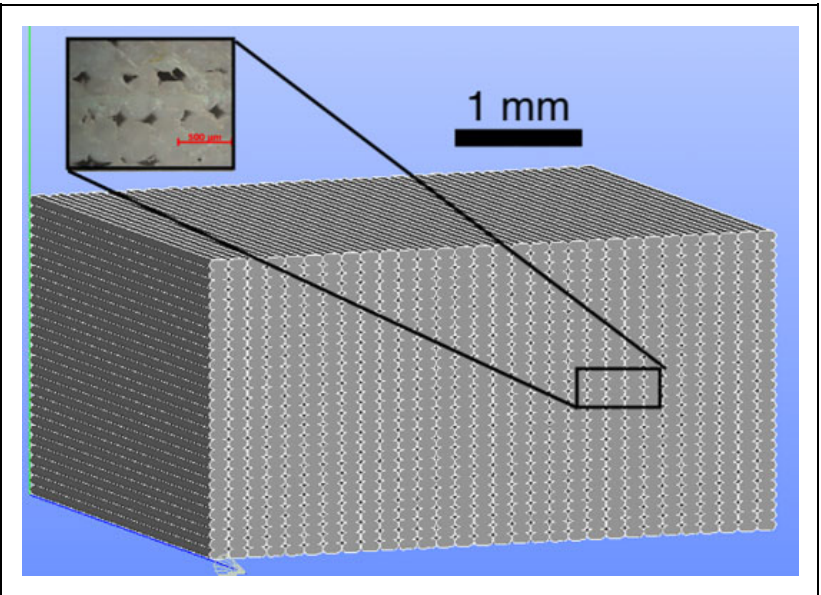

Figure 8. CAD model of standardized tensile specimen used for inverse analysis of transverse-isotropic, linear-elastic material model. Detail-view shows the cross-section of microstructure of an FDM-printed tensile specimen with a layer height of $0.2 \mathrm{~mm}$. CAD: computer-aided design; FDM: fused deposition modeling.

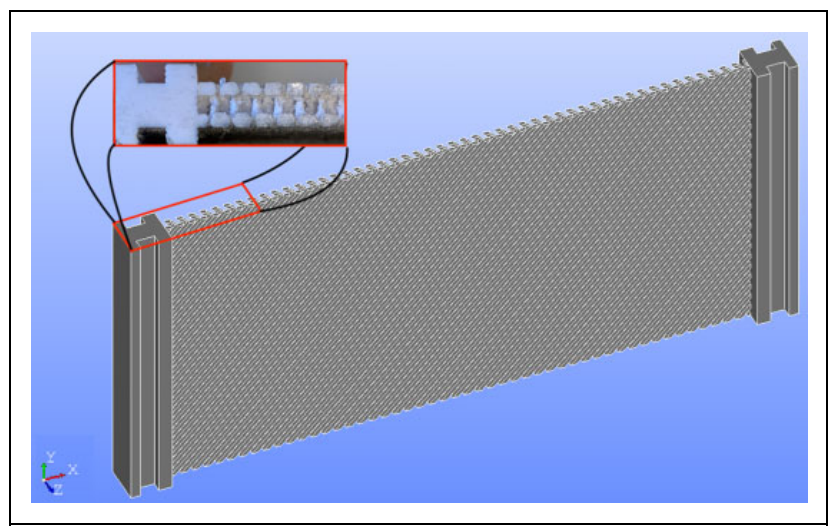

Figure 9. $C A D$ model of sample $B I$ by means of open-source software Salome 9.3. ${ }^{29}$ Red detail area in the left upper corner shows the transition from mounting toward the inner substructure. CAD: computer-aided design.

chosen because all computational homogenizations, and thereby, Young's moduli are obtained from energy calculations.

Numerical models are simulated by means of uniaxial tensile tests while Dirichlet boundary conditions are applied to the fixed mounting side and displacement is imposed along the lateral axis in the other mounting side. The gravitational forces have been neglected since the displacement is mainly influenced by surface loading conditions.

\section{Transverse isotropic material model and inverse analysis}

Transverse isotropic compliance matrix is given in Voigt's notation as follows 


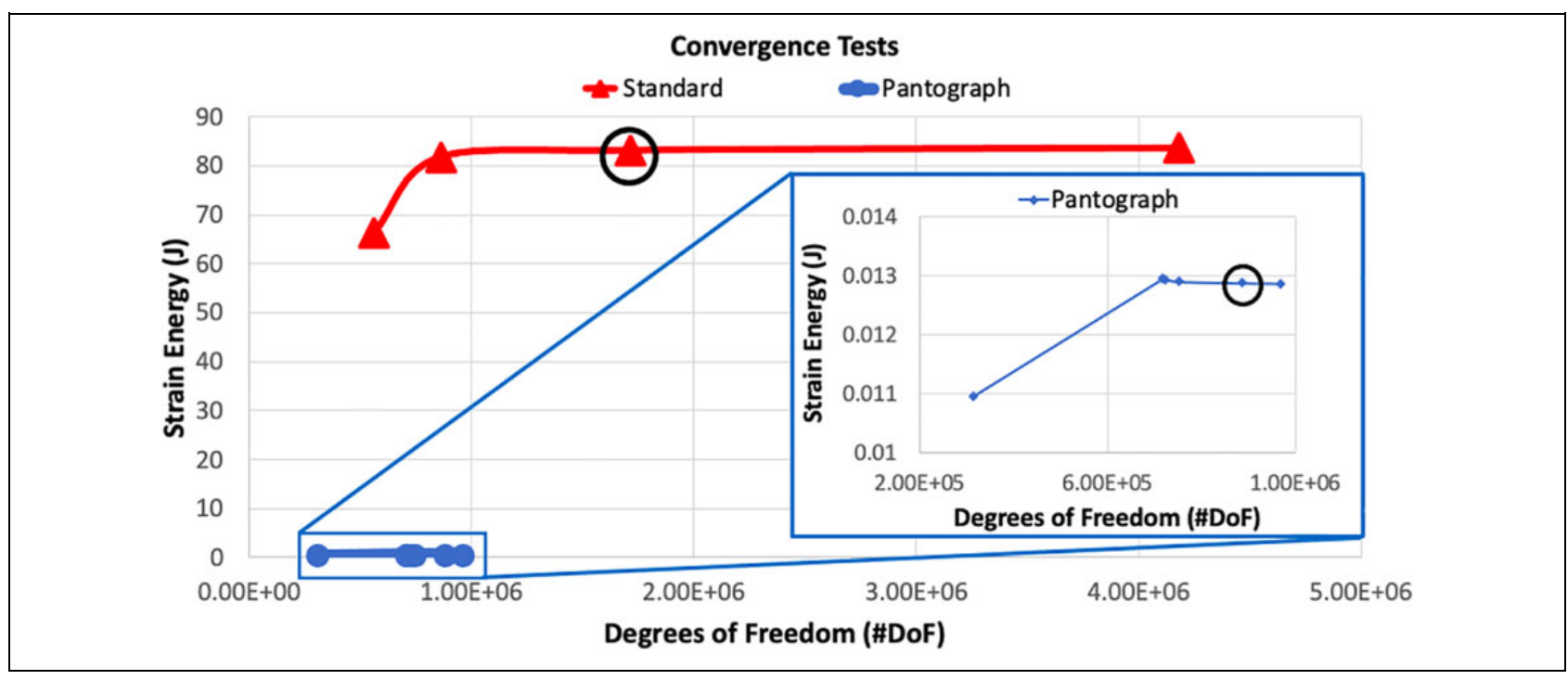

Figure 10. Convergence tests (exemplary) are shown for the standardized tensile specimen and the pantographic structure AI/A2. Strain energy is plotted over the number of degrees of freedom.

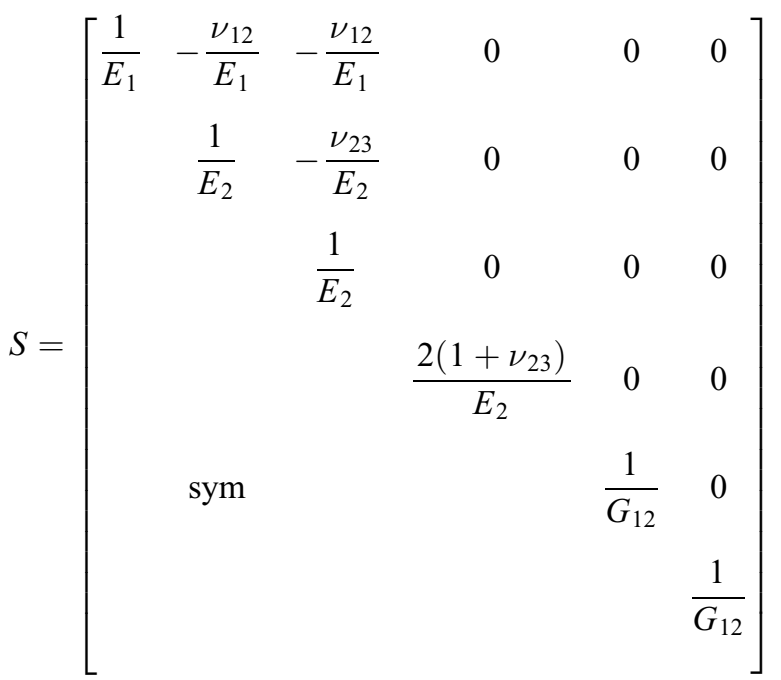

Six engineering constants, namely, Young's moduli $E_{1}$ and $E_{2}$, shear moduli $G_{12}$ and $G_{23}$, and Poisson's ratios $\nu_{12}$ and $\nu_{23}$, can be identified. To determine the so-called engineering constants, an inverse analysis is performed. Therefore, numerical simulations are carried out for three different cases, namely, case 1: with a filament orientation of $g=0^{\circ}$; case 2: with $g=90^{\circ}$; and case 3 : with $g=45^{\circ}$. To capture material response on the macroscale also, careful modeling at microscale is essential. For analysis performed on microscale, PETG is assumed to behave as a linear-elastic, isotropic material. Hence, Young's modulus $E=2150 \mathrm{MPa}$ and Poisson's ratio $\nu=0.35$ are given by the manufacturer. Engineering constants are determined from the simulations as follows:

1. To determine Young's modulus, $E_{1}$, CAD models with $g=0^{\circ}$ fiber orientations are employed (see Figure 8). We expect a linear displacement along the tensile direction, leading to constant strain, $\tilde{\varepsilon}_{11}=0.1$. Dirichlet boundary condition, $x_{1}=0$, is applied in one mounting side and set to $u_{1}=1$ in the other end. Strain energy is computed by equation $U=\frac{1}{2} \int \sigma_{i j} \varepsilon_{i j} \mathrm{~d} V$ leading to Young's modulus of $E_{1}=2 U\left(\tilde{\varepsilon}_{11}^{2} V\right)^{-1}$. Moreover, $\tilde{\varepsilon}_{22}$ is calculated by evaluating the transverse contraction of a standardized tensile specimen. This way it is possible to obtain the Poisson's ratio $\nu_{12}=-\frac{\tilde{\varepsilon}_{11}}{\tilde{\varepsilon}_{22}}$.

2. To calculate Young's modulus, $E_{2}$, CAD models with $g=90^{\circ}$ orientations are taken into account. Analogously, constant strain, $\tilde{\varepsilon}_{22}=0.1$, is assumed to be valid. Dirichlet boundary condition, $x_{2}=0$, is applied in one mounting side and set to $u_{2}=1$ in the other end. Young's modulus, $E_{2}$, is calculated analogously by reformulation of strain energy leading to $E_{2}=$ $2 U\left(\tilde{\varepsilon}_{22}^{2} V\right)^{-1} . \tilde{\varepsilon}_{33}$ is determined by transverse contraction leading to the Poisson's ratio of $\nu_{23}=-\frac{\tilde{\varepsilon}_{22}}{\tilde{\varepsilon}_{33}}$.

3. To determine the shear modulus, $G_{12}$, the Young's modulus, $\bar{E}$, for an fiber orientation of $g=45^{\circ}$ was calculated. $^{32}$ Again, constant strain, $\tilde{\varepsilon}_{11}=0.1$, is assumed to be valid and Dirichlet boundary condition, $x_{1}=0$, is applied to one mounting side and set to $u_{1}=1$ in the other end. CAD models with $g=45^{\circ}$ fiber orientations are employed because advantageous restrictions can be applied, ${ }^{30}$ resulting in $\bar{E}=2 U\left(\tilde{\varepsilon}_{11}^{2} V\right)^{-1}$. Hence, shear modulus, $G_{12}$, can be determined by

$$
G_{12}=\left(\frac{4}{\bar{E}}-\frac{1}{E_{1}}-\frac{1}{E_{2}}+\frac{2 \nu_{12}}{E_{1}}\right)^{-1}
$$

Determinations of the entire engineering constants result in the setup of the homogenized, transverse-isotropic 
Table 4. PETG's material parameters Young's modulus $E$ and Poisson's ratio $\nu$ are determined by means of linear elastic isotropic numerical simulation.

\begin{tabular}{lll}
\hline Slicing & Young's modulus & Poisson's ratio \\
\hline Strategy & $E_{2}=E_{90^{\circ}}=1408 \mathrm{MPa}$ & $\nu_{2}=\nu_{90^{\circ}}=0.35$ \\
A & $E_{1}=E_{0^{\circ}}=1882 \mathrm{MPa}$ & $\nu_{1}=\nu_{0}^{\circ}=0.35$ \\
B & $E_{2}=E_{90^{\circ}}=1408 \mathrm{MPa}$ & $\nu_{2}=\nu_{90^{\circ}}=0.35$ \\
C &
\end{tabular}

compliance-matrix in Voigt's notation (see "Results and discussion" section). Hence, this matrix can be used as input for the FEM simulations applied to PSs. Results of numerical calculations will be discussed and compared with results obtained from real experiments in "Results and discussion" section.

\section{Isotropic material model}

Young's moduli, $E_{1}$ and $E_{2}$, are determined by means of the aforementioned inverse analysis and computational homogenization procedure, which are described in detail in "Transverse isotropic material model and inverse analysis" section. Therefore, Young's moduli can also be interpreted as $E_{1}=E_{0^{\circ}}$ and $E_{2}=E_{90^{\circ}}$, respectively. Those parameters are used as inputs for the linearelastic, isotropic numerical simulations performed on PSs and are listed in Table 4.

To compare results of isotropic, linear-elastic numerical simulation with experimental results gained by means of an inverse analysis, parameters, $E_{g}$ (Young's modulus) and $\nu_{g}$, (Poisson's ratio) have to be determined. We make use of Hooke's law

$$
\sigma=E \varepsilon
$$

where the Young's modulus, $E$, which serves as a linear proportional factor between stress $\sigma$ and strain $\varepsilon$ is introduced. Stress $\sigma$ can be described as the quotient of measured force $\Delta F$ and the projection onto the mounting side of an initial cross-section $A_{\text {proj. }}$. Strain $\varepsilon$ is defined as the quotient of measured elongation $\Delta \ell$ and initial length $\ell_{0}$ resulting in the equation for Young's moduli

$$
E_{g}=\frac{\Delta F \ell_{0}}{\Delta \ell A_{\text {proj. }}}
$$

with $g=0^{\circ}$ for slicing strategy B and $g=90^{\circ}$ for slicing strategies A and C (see Table 4), respectively. In the case of an isotropic material, Poisson's ratio, $\nu_{g}$, is defined as the negative quotient of elongation in axial direction, $\varepsilon_{x x}$, and elongation in orthogonal direction, $\varepsilon_{y y}$, resulting in equation

$$
\nu_{g}=-\frac{\varepsilon_{x x}}{\varepsilon_{y y}}
$$

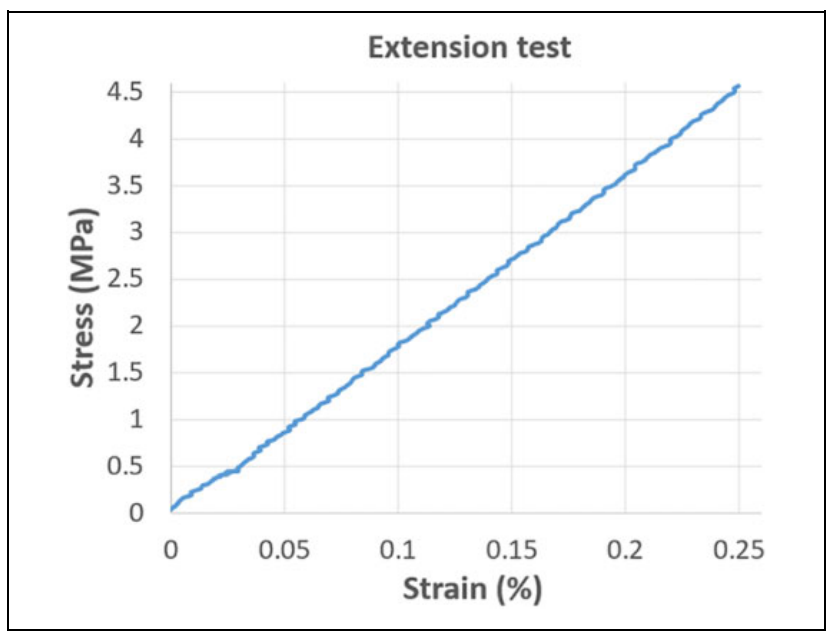

Figure I I. Stress-strain diagram of standardized extension test (according to Figure 5) applied to the standardized tensile specimen (see Figure 4).

Table 5. Comparison of Young's moduli of standardized tensile specimen obtained from real experiments and numerical simulations.

\begin{tabular}{lc}
\hline Characterization method & Young's modulus $\left(E_{1}=E_{0^{\circ}}\right)$ \\
\hline Standardized extension test & $1838 \mathrm{MPa}$ \\
Finite-element analysis & $1882 \mathrm{MPa}$ \\
\hline
\end{tabular}

\section{Results and discussion}

Results of extension tests performed on standardized tensile specimens and PSs are discussed separately. Furthermore, large and small deformations of PSs are debated. Finally, FEM simulations and small deformation results are compared.

\section{Standardized extension test}

Uniaxial, standardized extension tests were carried out to determine material parameters and to validate results obtained from the aforementioned computational homogenization. Engineering stress-strain relations of 3D-printed standardized tensile specimen are shown in Figure 11. Young's moduli obtained from experiments and FEM are listed in Table 5.

The values of Young's modulus obtained from experiments and FEM differ less than $2.33 \%$. These results present a proper accuracy and allow to trust the validated results of the inverse analysis based on FEM.

\section{Extension tests applied to pantographic structures}

Quasi-static, displacement-controlled extension tests were performed on all metamaterial samples. Displacement recorded by the device-own machine-code unit differs less than $\pm 1 \%$ from displacement calculated by DIC. 


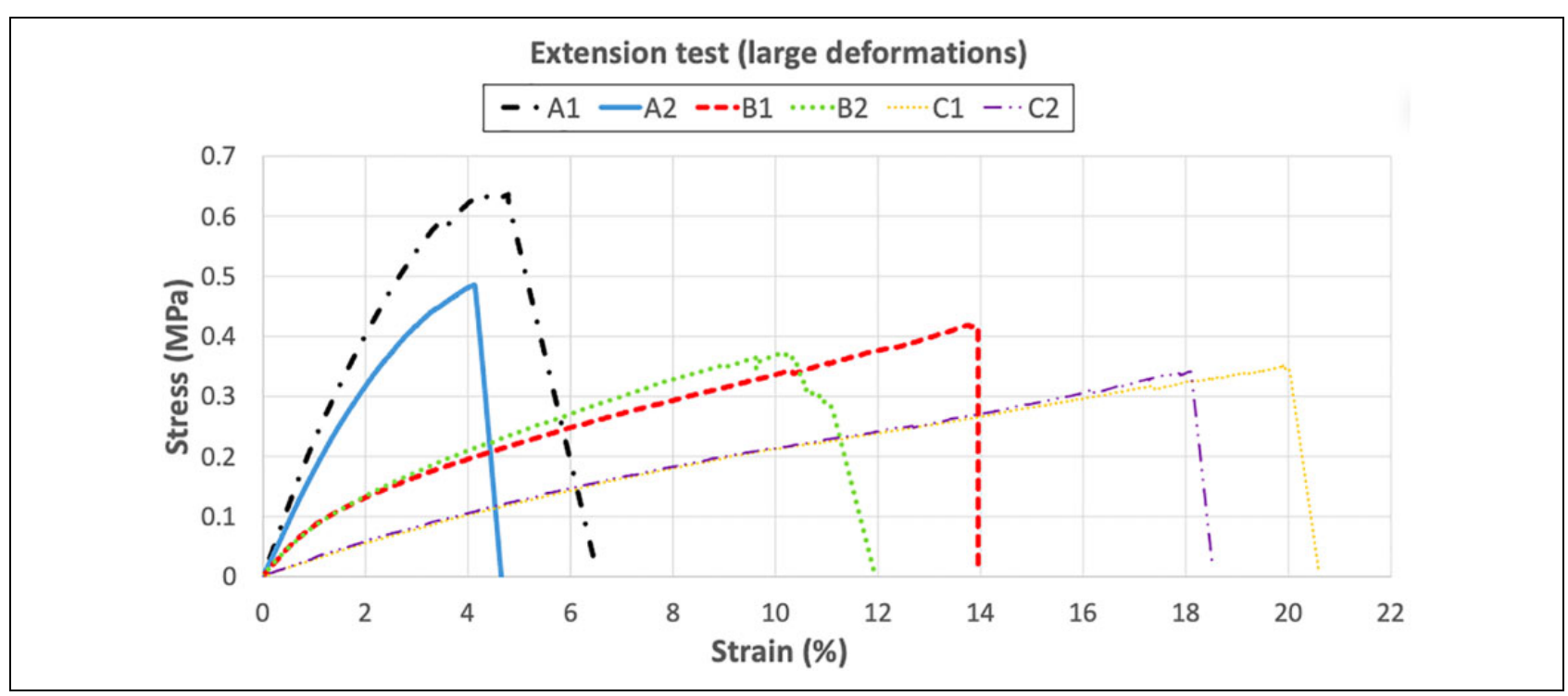

Figure I2. Stress-strain diagram of all six tested pantographs.

Therefore, the stress-strain diagrams are based on the translated displacement data obtained from the machine code. Extension load was applied to all specimens until ultimate failure of the whole metamaterial was achieved. Results of large deformations ( $\varepsilon_{x x}>2 \%$ axial elongation) and small deformations ( $\varepsilon_{x x} \leq 2 \%$ axial elongation) are going to be presented and discussed separately. Furthermore, experimental results of small deformations are going to be compared with linear-elastic results obtained from isotropic and transverse isotropic FEM simulations, respectively.

\section{Large deformations}

Deformation behavior of all samples gained in axial extension tests is shown in Figure 12. All specimens show nonlinear deformation behavior. They are able to deform at least $\varepsilon_{x x}=4 \%$ of axial elongation before ultimate failure occurs. Furthermore, all samples are even able to absorb much higher stresses after the first rupture (see Figure 7 for sample B1). Interestingly, specimen $\mathrm{C} 2$ shows (an almost linear elastic) deformation behavior up to about $\varepsilon_{x x}^{\mathrm{Cl}}=17.47 \%$ of axial elongation, where a first failure of the structure occurs. In contrast, specimen B1 shows a nonlinear inelastic deformation behavior up to about $\varepsilon_{x x}^{\mathrm{B} 1}=10.51 \%$ of axial elongation, where a first failure of the structure occurs without leading to ultimate rupture. Nevertheless, a smooth transition to plastic deformation takes place leading to a major constriction of the global structure (see Figure 7(b) and (c)). Because of the complex geometry, the reorganization of cylinders and beams results in a smart resilient material behavior. This is in good agreement with the results of tensile experiments applied to PSs made out polyamide noted in the literature. ${ }^{28}$ This peculiar, resilient observation was also mentioned for specimens made out of polyamide and aluminum investigated in shearing tests. ${ }^{8,14,33}$

Samples $\mathrm{C} 1$ and $\mathrm{C} 2$ show the highest elongation up to ultimate rupture $\left(\varepsilon_{x x}^{\mathrm{C} 1}=20 \%\right.$ and $\left.\varepsilon_{x x}^{\mathrm{C} 2}=18.1 \%\right)$. A main reason for this behavior is assumed to be caused by the combination of higher pivot height $(h=3 \mathrm{~mm})$ and smaller outer sample height $(l=35 \mathrm{~mm})$. This changes the boundary conditions resulting in a higher number of "free-hanging" beams, which are not connected to the mounting sides. Due to this fact, specimens $\mathrm{C} 1$ and $\mathrm{C} 2$ are able to deform much more in an elastic way. Furthermore, the higher pivot height $(h=3 \mathrm{~mm})$ in combination with slicing strategy $\mathrm{C}$ ( $g=90^{\circ}$ of fiber printing direction with respect to the lateral plane of the specimen), which was used during the manufacturing procedure for both samples $\mathrm{C} 1$ and $\mathrm{C} 2$, results in a more ductile deformation. Nevertheless, torsional loading conditions of the interconnected cylinders in the microscale may not result in an immediate failure of the structure because shear stresses are not as high as in the much stiffer samples A1 and A2. However, in contrast to specimens $\mathrm{B} 1$ and $\mathrm{B} 2$, slicing strategy $\mathrm{C}$ performed on samples $\mathrm{C} 1$ and $\mathrm{C} 2$ leads to periodic arrangements of cylinders and beams in the same direction, which is much more resistant against torque loading conditions on the substructure (e.g. see the top of Figure 13: axial printing direction along the axis of cylinders; see left of Figure 14: cylinders behave like slender beams). Therefore, alignments of printing layers in the pivots enable possibilities to deform in a more elastic regime (bending), for example, like in standard bending of Euler beams (see left of Figure 14).

Samples A1 and A2 show much stiffer/brittle deformation behavior than samples B1, B2, C1, and C2. Because of smaller cylinder height $(h=1 \mathrm{~mm})$ combined with slicing strategy A $\left(g=90^{\circ}\right)$, much stiffer deformation characteristic can be recognized. Weak bondings, voids, and 


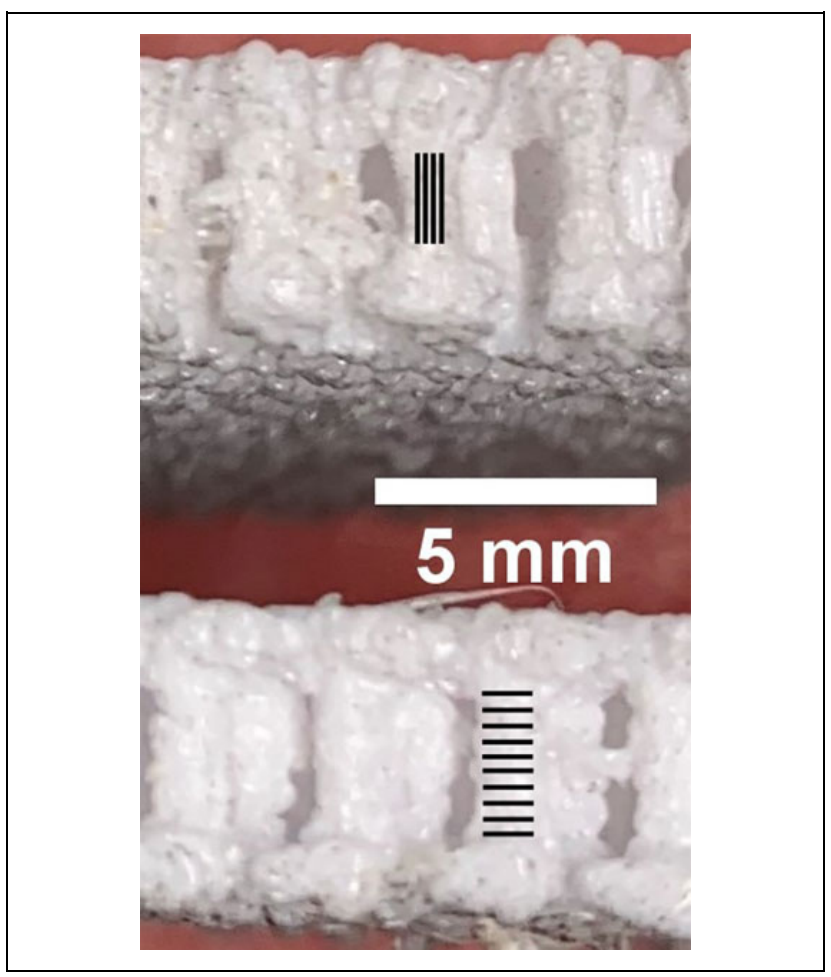

Figure 13. Microstructure of specimen $\mathrm{Cl}$ (top) printed with slicing strategy $C\left(g=90^{\circ}\right)$ and specimen $B I$ (bottom) printed with slicing strategy $B\left(g=0^{\circ}\right)$. Parallel lines indicate the printing directions.

misalignments between adjacent fibers and layers (see bottom in Figure 13, and detail-view in Figure 8, and misalignment in Figure 14) may also be further reasons why samples A1 and A2 show much more brittle behavior leading to smaller elongations. Furthermore, some leftovers of the support structure PVA in the pantographic substructure (especially in specimen A1 and A2 with smaller pivot heights) may be stiffen the material response during deformation in a non-negligible way (see voids in Figure 14).

These observations indicate that samples A1 and A2 are able to carry higher loads before breakage. From mechanical point of view, the resistance against torsion and bending of cylinders as well as the resistance against shear stresses in axial pivot/cylinder-layer direction result in a overall stiffer deformation behavior. In contrast, shearing stresses in the pivots of samples B1 and B2 with layer orientation of $g=0^{\circ}$ lead to a kind of compliant/resilient deformation behavior. The main reason is assumed to be caused by the higher pivot height of $h=3 \mathrm{~mm}$ in contrast to samples A1 and A2 $(h=1 \mathrm{~mm})$. Another reason may be that the maximal stresses are distributed into the beams in a much easier way. Summarized one may say that, on the one hand, samples A1 and $\mathrm{A} 2$ are able to resist higher extension loads (stresses) before the first rupture and ultimate failure occurs, but on the other hand, samples B1, B2, C1, and C2 are able to perform much higher axial elongation (strains) before ultimate failure happens.

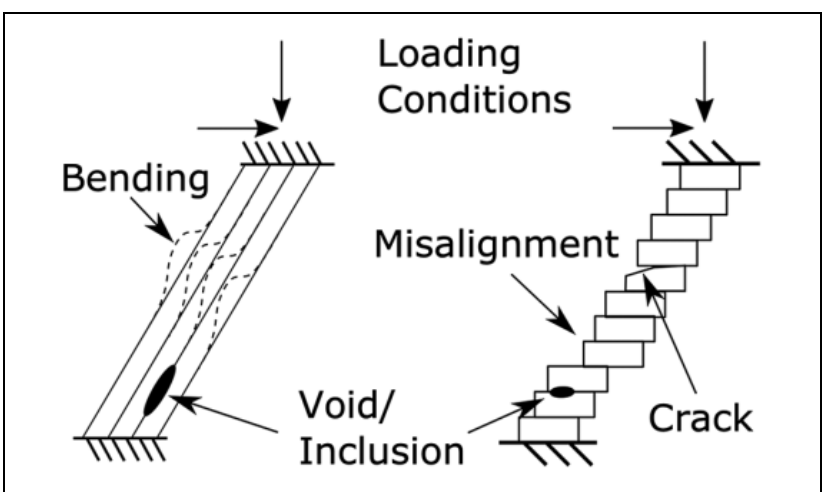

Figure 14. Schematics of deposited layers generated by means of different slicing strategies and possible manufacturing failures.

\section{Small deformations}

Figure 15 shows small deformation behavior of all six samples by means of stress-strain diagram. Interestingly, specimens B1 and $\mathrm{B} 2$ as well as specimens $\mathrm{C} 1$ and $\mathrm{C} 2$ show the same (almost linear) elastic deformation behavior with respect to each other. Samples A1 and A2 differ a lot from each other and show nonlinearity, especially, sample A2 follows a nonlinear inelastic regime. Due to the larger pivot heights $(h=3 \mathrm{~mm})$ of samples $\mathrm{B} 1, \mathrm{~B} 2, \mathrm{C} 1$, and $\mathrm{C} 2$, ductile deformation behavior with lower stiffness can be recognized in contrast to samples A1 and $\mathrm{A} 2$. The reason for relatively small elongations of $\mathrm{A} 1$ and $\mathrm{A} 2$ may be the very sensitive nature of PETG against humidity. These polymers can uptake even moisture from air.

\section{Experiments versus finite-element method}

The engineering constants are determined by an inverse analysis while tensile CAD models were prepared by means of microscope images, giving us important knowledge about the arrangements of microstructural features such as fibers and voids (e.g. see detail-view in Figure 8). Hence, material models include important microstructural characteristics of FDM polymers and have been prepared in accordance to recommendations of the literature. ${ }^{18,30}$ For the configuration listed in Table 1, the transverse isotropic stiffness matrix in Voigt's notation reads

$$
C=\left[\begin{array}{cccccc}
2144 & 374 & 374 & 0 & 0 & 0 \\
374 & 1935 & -864 & 0 & 0 & 0 \\
374 & -864 & 1935 & 0 & 0 & 0 \\
0 & 0 & 0 & 1399 & 0 & 0 \\
0 & 0 & 0 & 0 & 545 & 0 \\
0 & 0 & 0 & 0 & 0 & 545
\end{array}\right] \mathrm{MPa}
$$

This stiffness matrix is used as input for the linearelastic, transverse isotropic numerical simulation of PS. Young's moduli, $E_{1}$ and $E_{2}$, calculated by inverse analysis are also used as input parameters in the linear-elastic, isotropic simulation of PS and are given in Table 6. 


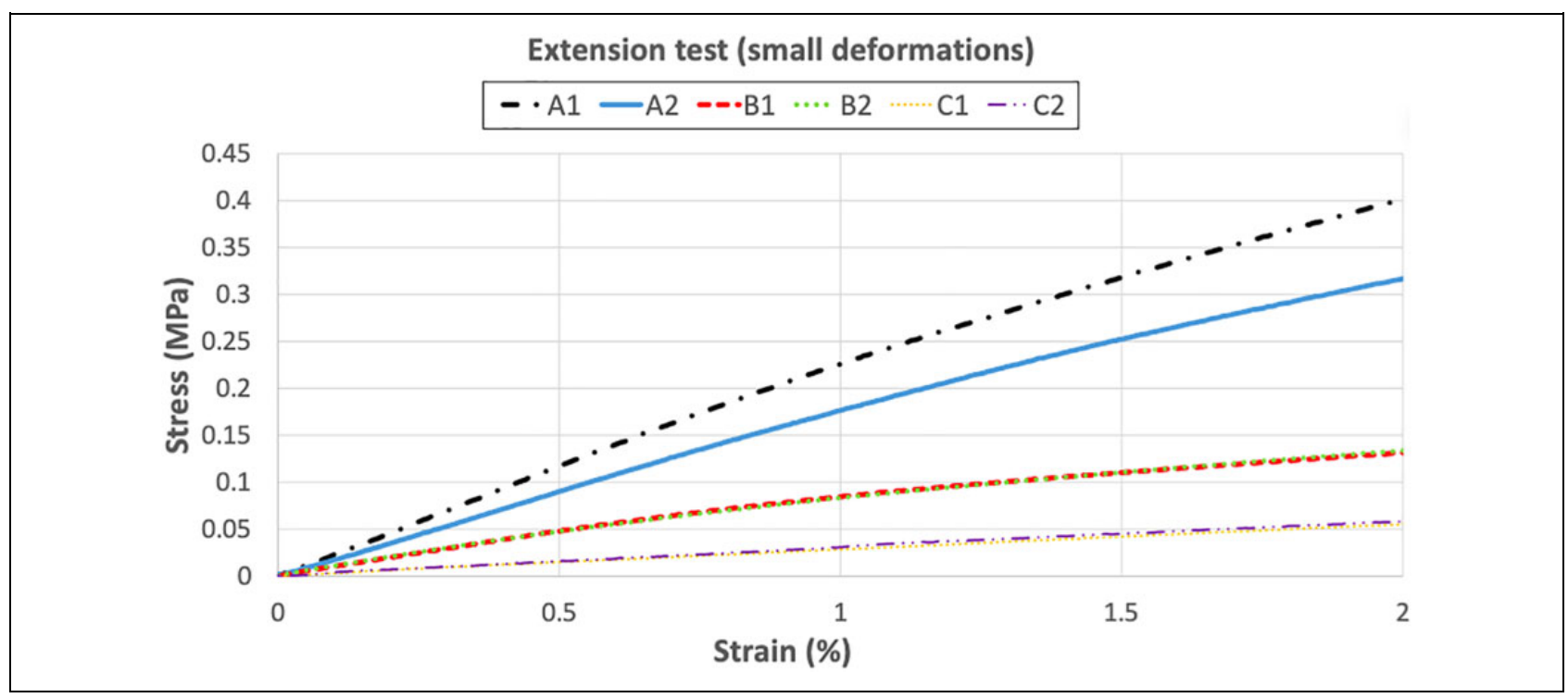

Figure 15. Stress-strain diagram of all investigated specimens AI, A2, BI, B2, CI, and C2 for small deformations.

Table 6. Established material/engineering parameters of the linear-elastic, isotropic model: Young's moduli $E_{0^{\circ}}\left(=E_{1}\right)$ and $E_{90^{\circ}}\left(=E_{2}\right)$, and Poisson's ratios $\nu_{0^{\circ}}\left(=\nu_{1}\right)$ and $\nu_{90^{\circ}}\left(=\nu_{2}\right)$.

\begin{tabular}{lrr}
\hline Layer orientation & Young's modulus & Poisson's ratio \\
\hline $0^{\circ}$ & $E_{0^{\circ}}=1882 \mathrm{MPa}$ & $\nu_{0}^{\circ}=0.35$ \\
$90^{\circ}$ & $E_{90^{\circ}}=1408 \mathrm{MPa}$ & $\nu_{90^{\circ}}=0.35$ \\
\hline
\end{tabular}

The calculated deformation profile based on specimen B1 is given at the top of Figure 16 and is compared with 2D DIC evaluation for an axial elongation of $\varepsilon_{x x}^{\mathrm{B} 1}=4 \%$ in the bottom of Figure 16. Both show maximal vertical displacements of about $\Delta_{d y}=2.5 \mathrm{~mm}$ and are in good agreement to each other.

Young's moduli gained from experimental characterizations and Young's moduli obtained from FEM simulations are compared in Table 7. Obviously, values of the transverse isotropic model fit the experimentally measured parameters in a much better way in contrast to the isotropic material model.

Furthermore, results of transverse isotropic FEM simulations are compared with results obtained from extension tests by means of stress-strain curves in Figure 17 . We state that transverse isotropic model is sufficient enough to capture the deformation behavior of pantographic metamaterials for axial elongations up to about $\varepsilon_{x x}=1.2 \%$. Isotropic model fails as there is an arithmetic difference of about 18-19\% to experimental results. According to process parameters, all specimens have the same layer orientation leading to UD structures. After comparative analysis between different material models and experimental results, we observe that this UD orientation leads to transversal isotropy despite the pantographics complex microstructure. These outcomes indicate the importance of the selection of process parameters in FDM.

All microstructural features (such as fibers, voids, and their interfaces) were modeled homogeneously. But this

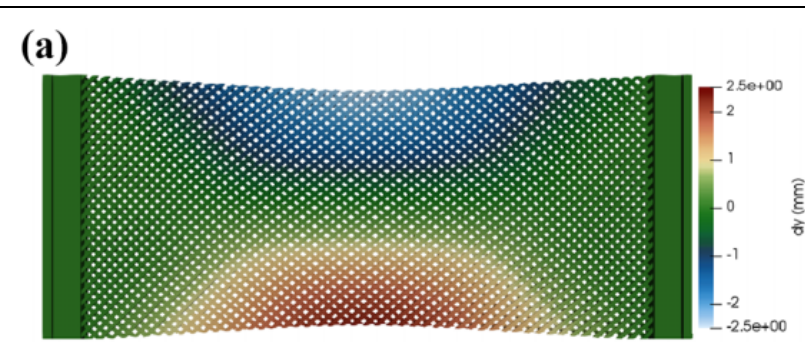

(b)

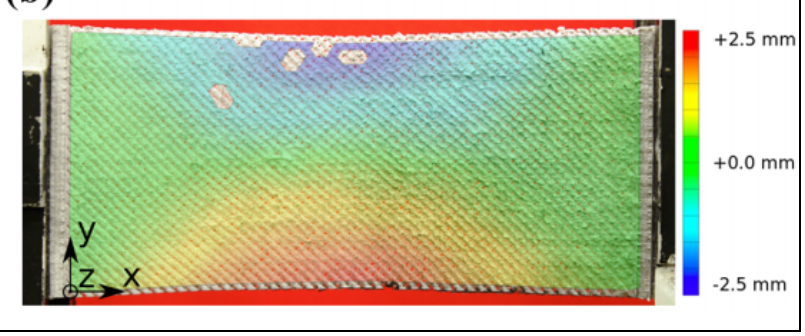

Figure 16. Transverse isotropic FEA simulation of specimen BI validated by $2 \mathrm{D}$ DIC: comparison of vertical displacements of results gained from the experiment by means of DIC (b) with numerical results calculated by means of FEniCS simulation ${ }^{17}$ (a) of sample $B I$ at about $\varepsilon_{x x}^{B I}=4 \%$ of axial elongation during extension test. Both graphs show a maximal vertical displacement of about $\Delta_{d y}= \pm 2.5 \mathrm{~mm}$ and are in good agreement to each other. DIC: digital image correlation; FEA: finite-element analysis.

homogeneity does not exist in the real manufactured specimens. Due to manufacturing circumstances in FDM printing, heterogeneous inner structures occur. Temperature differences between solidified fibers and deposited melt cause heterogeneous interfaces, which were not taken into account in FEM simulations. This could also explain the arithmetic difference of about $6 \%$ between transverse isotropic FEM and experimental results. 
Table 7. Comparison/validation of Young's moduli gained by linear-elastic, isotropic simulation, and by linear-elastic, transverse isotropic simulation, and by experiments.

\begin{tabular}{lcc}
\hline Sample & $\mathrm{Al}$ & $\mathrm{A} 2$ \\
\hline E-Modul/experimental & $22.84 \mathrm{MPa}$ & $18.20 \mathrm{MPa}$ \\
E-Modul/isotrop & $16.40 \mathrm{MPa}$ & $16.44 \mathrm{MPa}$ \\
E-Modul/transverse isotrop & $20.05 \mathrm{MPa}$ & $20.05 \mathrm{MPa}$ \\
Error/isotrop & $28.19 \%$ & $9.67 \%$ \\
Error/transverse isotrop & $12.20 \%$ & $9.22 \%$ \\
\hline Sample & $\mathrm{BI}$ & $\mathrm{B} 2$ \\
\hline E-Modul/experimental & $9.58 \mathrm{MPa}$ & $9.81 \mathrm{MPa}$ \\
E-Modul/isotrop & $8.45 \mathrm{MPa}$ & $8.45 \mathrm{MPa}$ \\
E-Modul/transverse isotrop & $9.20 \mathrm{MPa}$ & $9.20 \mathrm{MPa}$ \\
Error/isotrop & $11.81 \%$ & $13.90 \%$ \\
Error/transverse isotrop & $3.90 \%$ & $6.22 \%$ \\
\hline Sample & $\mathrm{Cl}$ & $\mathrm{C} 2$ \\
\hline E-Modul/experimental & $3.42 \mathrm{MPa}$ & $3.44 \mathrm{MPa}$ \\
E-Modul/isotrop & $2.57 \mathrm{MPa}$ & $2.57 \mathrm{MPa}$ \\
E-Modul/transverse isotrop & $3.35 \mathrm{MPa}$ & $3.35 \mathrm{MPa}$ \\
Error/isotrop & $24.85 \%$ & $25.29 \%$ \\
Error/transverse isotrop & $2.20 \%$ & $2.61 \%$ \\
\hline
\end{tabular}

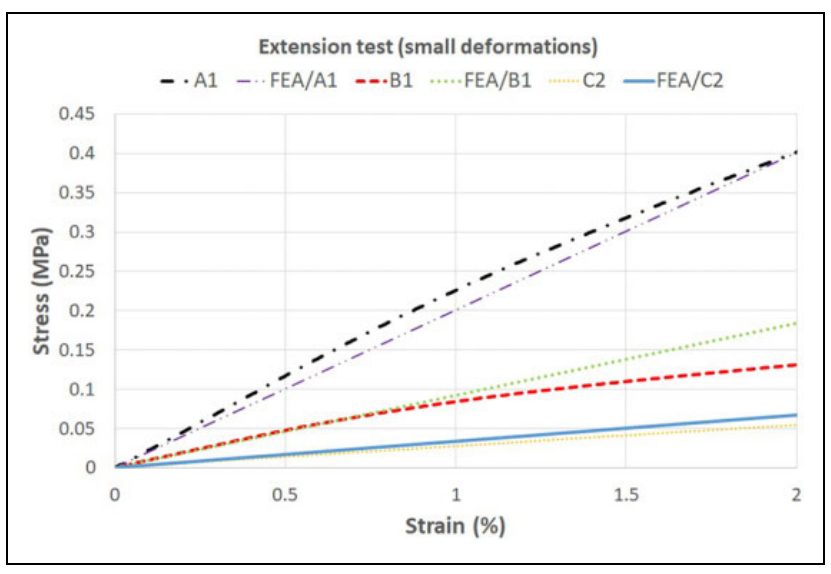

Figure 17. Comparison of stress-strain relations obtained from extension tests performed on specimens $\mathrm{AI}, \mathrm{BI}$, and $\mathrm{C} 2$ and FEM calculations (transverse isotropic linear-elastic FEM simulation by means of FEniCS ${ }^{17}$ ). Computationally homogenized transverse isotropic stiffness matrix in Voigt's notation has been used as FEM-input parameter. FEM: finite-element method.

\section{Conclusion}

Three types of pantographic metamaterials consisting of different substructures were additively manufactured (namely, FDM) using three different slicing strategies. PETG was used as filament material. Young's modulus of 3D-printed PETG was determined as $E_{1}=1838 \mathrm{MPa}$, according to ISO 527-2. Furthermore, six quasi-static, uniaxial extension tests have been performed onto three differently sized pantographic specimen batches. The 2D DIC was used to investigate the material/deformation behavior qualitatively and quantitatively. Linear and nonlinear deformations were taken into account. Inverse analysis has been performed resulting in the determination of engineering/material parameters like Young's modulus and Poisson's ratio. Additionally, isotropic and transverse isotropic linear-elastic FEM simulations have been carried out and have been validated with experimental results. Transverse isotropic simulations are in good agreement with small deformation experiments up to about $\varepsilon_{x x}=1.2 \%$ of axial elongation.

In general, $3 \mathrm{D}$-printed metamaterials with a pantographic substructure show much higher strains $\left(\varepsilon_{x x}>>3.5 \%\right)$ than $3 \mathrm{D}$-printed standardized tensile specimens with more than 20 layers (see the literature ${ }^{30}$; highest strains at about $\varepsilon_{x x}=3.5 \%$ of axial elongation). PSs manufactured using slicing strategy B (layer orientation of $0^{\circ}$ with respect to the lateral plane of the metamaterial) show a high elastic and therefore resilient deformation behavior resulting in about $\varepsilon_{x x}=9-15 \%$ of axial elongation. Furthermore, PSs printed with slicing strategy $\mathrm{C}$ (layer orientation of $90^{\circ}$ with respect to the lateral plane of the metamaterial) show an even higher axial elongation up to about $\varepsilon_{x x}=18-20 \%$. In contrast, structures printed with a layer orientation of $90^{\circ}$ (slicing strategy A) and smaller inner parameters on the substructure are only able to deform to about $\varepsilon_{x x}=4-5 \%$ of axial elongation. The big advantage of pantographic metamaterials is the high resilient deformation behavior of the whole structure against ultimate failure even after local ruptures pop up. Hence, the combination of these peculiar mechanical properties like large (elastic) deformations or adjustable stiffnesses renders those metamaterials to be very useful for technical applications in lightweight or medical industries, respectively.

\section{Acknowledgment}

We acknowledge support by the German Research Foundation and the Open Access Publication Fund of TU Berlin.

\section{Author contributions}

The authors AÖ and GG contributed equally to this work.

\section{Declaration of conflicting interests}

The author(s) declared no potential conflicts of interest with respect to the research, authorship, and/or publication of this article.

\section{Funding}

The author(s) received no financial support for the research, authorship, and/or publication of this article.

\section{ORCID iD}

Gregor Ganzosch (D) https://orcid.org/0000-0003-0430-8830

\section{References}

1. Gibson LJ and Ashby MF. Cellular solids: structure and properties. Cambridge: Cambridge University Press, 1999.

2. Barchiesi E, Spagnuolo M and Placidi L. Mechanical metamaterials: a state of the art. Math Mech Solids 2019; 24(1): 212-234. 
3. Placidi L, Greco L, Bucci S, et al. A second gradient formulation for a 2D fabric sheet with inextensible fibres. Zeitschrift für angewandte Mathematik und Physik 2016; 67(5): 114.

4. dell'Isola F, Seppecher P, Alibert JJ, et al. Pantographic metamaterials: an example of mathematically driven design and of its technological challenges. Continuum Mech Therm 2019; 31(4): 851-884.

5. dell'Isola F, Seppecher P, Spagnuolo M, et al. Advances in pantographic structures: design, manufacturing, models, experiments and image analyses. Continuum Mech Therm 2019; 31(4): 1231-1282.

6. dell'Isola F, Spagnuolo M, Barchiesi E, et al. 3D pantographic metamaterial: a (not so) particular case. In: dell'Isola F and Steigmann DJ (eds) Discrete and continuum models for complex metamaterials. Cambridge: Cambridge University Press, 2020, p. 103.

7. dell'Isola F, Giorgio I, Placidi L, et al. Pantographic metamaterials: a view towards applications. Mater Phys Mech 2019; 42(5): 637-645.

8. Yang H, Ganzosch G, Giorgio I, et al. Material characterization and computations of a polymeric metamaterial with a pantographic substructure. Zeitschrift für angewandte Mathematik und Physik 2018; 69(4): 105.

9. Barboura S and Li J. Establishment of strain gradient constitutive relations by using asymptotic analysis and the finite element method for complex periodic microstructures. Int $J$ Solids Struct 2018; 136: 60-76.

10. dell'Isola F, Giorgio I, Pawlikowski M, et al. Large deformations of planar extensible beams and pantographic lattices: heuristic homogenization, experimental and numerical examples of equilibrium. Proc Royal Soc A 2016; 472(2185): 20150790.

11. Seppecher P, Alibert JJ and dell'Isola F. Linear elastic trusses leading to continua with exotic mechanical interactions. $J$ Phys Conf Ser 2011; 319: 12-18.

12. Alibert JJ, Seppecher P and dell'Isola F. Truss modular beams with deformation energy depending on higher displacement gradients. Math Mech Solids 2003; 8(1): 51-73.

13. Placidi L, Andreaus U and Giorgio I. Identification of twodimensional pantographic structure via a linear $\mathrm{d} 4$ orthotropic second gradient elastic model. J Eng Math 2016; 103(1): 1-21.

14. Ganzosch G, Hoschke K, Lekszycki T, et al. 3Dmeasurements of 3D-deformations of pantographic structures. Tech Mech 2018; 38(3): 233-245.

15. Placidi L, Rosi G and Barchiesi E. Analytical solutions of 2dimensional second gradient linear elasticity for continua with cubic-d4 microstructure. In: Eremeyev VA, Echsner A, dell'Isola F, et al. (eds) New achievements in continuum mechanics and thermodynamics. Springer Nature Switzerland AG, 2019, pp. 383-401.

16. Placidi L, Barchiesi E and Della Corte A. Identification of twodimensional pantographic structures with a linear $\mathrm{d} 4$ orthotropic second gradient elastic model accounting for external bulk double forces. In: dell'Isola F, Steigmann D and Sofonea M (eds) Mathematical modelling in solid mechanics. Springer Nature Singapore Pvt. Ltd. 2017, pp. 211-232.
17. Fenics Project. https://fenicsproject.org/. (accessed 15 May 2021).

18. Kiendl $\mathrm{J}$ and Gao C. Controlling toughness and strength of FDM 3D-printed PLA components through the raster layup. Compos B Eng 2020; 180: 107562.

19. Özen A, Auhl DW, Völlmecke C, et al. Optimization of manufacturing parameters and tensile specimen geometry for fused deposition modeling (FDM) 3D-printed PETG. Materials 2021; 14(10): 2556.

20. Rahali Y, Giorgio I, Ganghoffer J, et al. Homogenization à la piola produces second gradient continuum models for linear pantographic lattices. Int J Eng Sci 2015; 97: 148-172.

21. Barchiesi E, Ganzosch G, Liebold C, et al. Out-of-plane buckling of pantographic fabrics in displacement-controlled shear tests: experimental results and model validation. Continuum Mech Therm 2018; 31: 1-13.

22. Giorgio I, Rizzi NL, Andreaus U, et al. A two-dimensional continuum model of pantographic sheets moving in a $3 \mathrm{D}$ space and accounting for the offset and relative rotations of the fibers. Math Mech Complex Syst 2019; 7(4): 311-325.

23. De Angelo M, Barchiesi E, Giorgio I, et al. Numerical identification of constitutive parameters in reduced-order bidimensional models for pantographic structures: application to out-of-plane buckling. Arch Appl Mech 2019; 89(7): 1333-1358.

24. Boutin C, Giorgio I, Placidi L, et al. Linear pantographic sheets: asymptotic micro-macro models identification. Math Mech Complex Syst 2017; 5(2): 127-162.

25. Giorgio I. Numerical identification procedure between a micro-Cauchy model and a macro-second gradient model for planar pantographic structures. Zeitschrift für angewandte Mathematik und Physik 2016; 67(4): 95.

26. Turco E, Barcz K, Pawlikowski M, et al. Non-standard coupled extensional and bending bias tests for planar pantographic lattices. Part I: numerical simulations. Zeitschrift für angewandte Mathematik und Physik 2016; 67(5): 122.

27. Turco E, Dell'Isola F, Cazzani A, et al. Hencky-type discrete model for pantographic structures: numerical comparison with second gradient continuum models. Zeitschrift für angewandte Mathematik und Physik 2016; 67(4): 85.

28. Juritza A, Yang H and Ganzosch G. Qualitative investigations of experiments performed on 3D-FDM-printed pantographic structures made out of PLA. Cham: Springer International Publishing, 2019, pp. 197-209.

29. Salome-Platform. https://www.salome-platform.org/ (accessed 15 May 2021).

30. Özen A, Auhl DW, Völlmecke C, et al. Exploring the role of manufacturing parameters on microstructure and mechanical properties of fused deposition modeling (FDM) polymers. Appl Compos Mater (submitted). Springer

31. Chang KH. Chapter 7 - Structural analysis. In: Chang KH (ed) e-Design. Boston: Academic Press, 2015, pp. 325-390.

32. Jones RM. Mechanics of composite materials. Milton Park: Taylor \& Francis, 1999.

33. Ganzosch G, dell'Isola F, Turco E, et al. Shearing tests applied to pantographic structures. Acta Polytech CTU Proc 2017; 7: 1-6. 